\title{
TRUTH AS VALUE AND DUTY: LESSONS OF MATHEMATICS ${ }^{1}$
}

\author{
Yu. I. Manin \\ Max-Planck-Institut für Mathematik, Bonn, Germany, \\ and Northwestern University, Evanston, USA
}

\section{Introduction}

Imagine that you open your morning newspaper and read the following report:

Brownsville, AR. A local object partially immersed in a liquid was buoyed upward Tuesday by a force equal to the weight of the liquid displaced by that object, witnesses at the scene reported. As of press time, the object is still maintaining positive buoyancy.

In fact, I did read this report in the ONION; I have only abridged it to add a Fénéonian touch.

If this meeting had been dedicated to the nature of the comical, one could produce an interesting analysis of the clever silliness of this parody. But as we are preoccupied with truth, I will use it in order to illustrate the differences between the attitudes to truth among practitioners of social sciences and law (as exemplified by $[\mathrm{BoHa}]$ ) on the one hand, and that of, say, physicists, on the other.

To put it crudely, in social sciences information comes from witnesses; but in what sense was Archimedes' role in his discovery that of a witness, and are the experimental observations generating/supporting a physical theory on an equal footing with the observations of witnesses to a crime scene, or respondents to a poll?

Now, imagine another report, that could have been posted on the web-site of the Department of Physics of Cambridge University:

The Cavendish Laboratory News $\&$ Features bulletin announced yesterday that a Cavendish student has won Science, Engineering and Technology award. He managed to measure the constant $\pi$ with unprecedented precision: $\pi=3,1415925 \ldots$ with an error \pm 2 at the last digit.

\footnotetext{
${ }^{1}$ Talk at the International Symposium of the Balzan Foundation "Truth in the Humanities, Science and Religion", Lugano, May 16-17, 2008.
} 
I must confess right away that I did not read but simply fabricated this spoof in order to stress the further differences between the attitudes towards truth, now held by physicists and by mathematicians respectively.

On the one hand, formally such an announcement would make perfect sense: the mathematical constant $\pi$ can be measured with some precision, in the same way that any physical constant such as the speed of light $c$, or the mass of the electron can be measured. The maximum achievable precision, at least of a "naive" direct measurement of $\pi$, is determined by the degree to which we can approximate ideal Euclidean rigid bodies by real physical ones. The limits to this approximation are set by the atomic structure of matter, and in the final analysis, by quantum effects.

On the other hand, in order to get in principle as many digits of $\pi$ as one wishes, measurements are not required at all. Instead, one can use one of the many existing formulas/algorithms/software codes and do it on a sheet of paper, a pocket calculator, or a supercomputer. This time the limits of precision are determined by the physical limitations of our calculator: the size of the sheet of paper, memory of computer, construction of the output device, available time ...

What I want to stress now is that $\pi$ imagined as an infinite sequence of its digits, is not amenable to a "finite" calculation: even the number of digits of $\pi$ equal to the number of atoms in the observable Universe, would not exhaust $\pi$. Nevertheless, mathematicians speak about $\pi$ and work with $\pi$ as if it were a completely well defined entity, graspable in its entirety not only by one exceptional super-Mind, but by the minds of all trained researchers, never doubting that when they speak of $\pi$, they speak about one and the same ideal object, as rigid as if it really exists in some Platonic world.

In fact, one facet of this rigidity can be expressed by a few theorems implying that whatever exact formula, algorithm, or software code we might use to calculate $\pi$ and whatever precision we choose, we will always get the same result. If we do not, either our formula was wrong, or the calculator made a mistake/there was a bug in the code/output device could not cope with the quantity of information ...

Contemplating this example, we may grasp the meaning of the succinct description of mathematics by Davis and Hersh ([DaHe]): "the study of mental objects with reproducible properties".

However, I want to use this example in order to stress that most of the deep mathematical truths are about infinity and infinitary mental constructs rather than experimentally verifiable finitary - and finite - operations, that can be modeled using actual objects of the physical world. 
... mais je ne le crois pas!

G. Cantor to R. Dedekind, June 29, 1877

Before Georg Cantor, infinity appeared in mathematical theorems mostly implicitly, through the quantifier "all" (which also could be only implicit as in most Euclid's theorems).

Cantor proved the first theorem ever in which infinities themselves were objects of consideration. Slightly modernizing his arguments, we can say that he invented two or three mental constructions allowing us to compare sizes (technically, cardinalities) of infinite (in fact, finite as well) sets:

a) Two sets $X, Y$ have equal cardinalities, symbolically $|X|=|Y|$, if their elements $x \in X, y \in Y$ can be joined in pairs $(x, y)$ in such a way that each $x$ is paired with exactly one $y$ and each $y$ with exactly one $x$.

b) The cardinality of $X$ is called "less or equal" to that $Y$, symbolically $|X| \leq|Y|$, if there is a subset $X^{\prime} \subset Y$ such that $|X|=\left|X^{\prime}\right|$.

After these two definitions, the famous Cantor's theorem can be proved in several lines:

c) The set of all subsets of $X$, symbolically $P(X)$, has cardinality strictly larger than that of $X$.

Since we may iterate this construction, forming consecutively $P(P(X)), P(P(P(X)))$, $\ldots$, we see that there exists an infinite scale of infinities of growing sizes.

The proof of c) consists of two remarks. The first one says that $|X| \leq|P(X)|$, because $X$ can be in a tautological way paired with a part of $P(X)$ consisting of one-element subsets of $X$.

The second remark is (a remake of) the famous Cantor's diagonal argument, using reductio ad absurdum. Imagine that $|X|=|P(X)|$. Then we can pair each $x \in X$ with some $S_{x} \subset X$ in such a way that any subset $S \subset X$ has the form $S_{y}$ for some $y \in S$. Choose such a pairing (technically, one-to-one correspondence). Define

$$
S=\text { the set of all } x \text { such that } x \notin S_{x} .
$$

This $S$ must be of the form $S_{y}$ for some $y \in X$, but then both logical possibilities, $y \notin S_{y}$ and $y \in S_{y}$ lead to a contradiction, so that the postulated one-to-one correspondence cannot exist. 
Of course, the last key argument goes back to the ancient "liar's paradox". It was revived again in a different context in the 20th century by Tarski and Gödel. Tarski's theorem features the ominous, at least for the purposes of this conference, name "inexpressibility of truth".

In the final analysis, self-referentiality was used to produce several deep mathematical arguments, and this became possible only when the mathematical universe became so extended that the language of mathematics could be embedded into this universe as a part of it. In particular, Leibniz's dream of merging language with meta-language became a reality.

\section{3}

The best test of truth is the power of the thought to get itself accepted in the competition of the market.

Justice Oliver Wendell Holmes, Jr (1919)

When Cantor first presented his diagonal argument in a letter to Dedekind in 1873 , it was worded differently and used only to prove that the cardinality of the natural numbers is strictly less than that of the real numbers. The discovery of the proof itself was in a sense hardly more important than the discovery of the definition of what it means, for one infinity to be larger than another one.

As soon as this was achieved, Cantor started thinking about the cardinality of the reals compared with that of the pairs of reals, or, geometrically, sets of points of a curve and of a surface respectively. They turned out to be equal! If we have a pair of numbers $(\alpha, \beta)$ in $(0,1)$, Cantor suggested to produce from them the third number $\gamma \in(0,1)$ by putting decimal digits of $\alpha$ to the odd places, and those of $\beta$ to the even places. One sees, that vice versa, $(\alpha, \beta)$ can be reconstructed from $\gamma$. Dedekind, who was informed by Cantor's letter about this discovery as well, remarked that this does not quite work because some rational numbers have two decimal representations, such as $0,499999 \cdots=0,5000000 \ldots$ Cantor had to spend some time to amend the proof, but this was a minor embarrassment, in comparison with the fascinating novelty of the fact itself: "Ce que je vous ai communiqué tout récemment est pour moi si inattendue, si nouveau, que je ne pourrai pour ainsi dire pas arriver à une certaine tranquillité d'esprit avant que je n'aie reçu, très honoré ami, votre jugement sur son exactitude. Tant que vous ne m'aurez pas approuvé, je ne puis que dire: je le vois, mais je ne le crois pas", as Cantor famously wrote to Dedekind.

This returns us to the basic question on the nature of truth. 
We are reminded that the notion of "truth" is a reification of a certain relationship between humans and texts/utterances/statements, the relationship that is called "belief", "conviction" or "faith", and which itself should be analyzed, together with other primary notions invoked in this definition.

Professor Blackburn in [Bl] extensively discussed other relationships of humans to texts, such as scepticism, conservatism, relativism, deflationism. However, in the long range all of them are secondary in the practice of a researcher in mathematics.

So I will return to truth.

I will skip analysis of the notion of "humans" :=) and will only sketch what must be said about texts, sources of conviction, and methods of conviction peculiar to mathematics.

Texts. Alfred North Whitehead allegedly said that all of Western philosophy was but a footnote to Plato.

The underlying metaphor of such a statement is: "Philosophy is a text", the sum total of all philosophic utterances.

Mathematics decidedly is not a text, at least not in the same sense as philosophy. There are no authoritative books or articles to which subsequent generations turn again and again for wisdom. Except for historians, nobody reads Euclid, Newton, Leibniz or Hilbert in order to study geometry, calculus or mathematical logic. The life span of any mathematical paper or book can be years, in the best (and exceptional) case decades. Mathematical wisdom, if not forgotten, lives as an invariant of all its (re)presentations in a permanently self-renewing discourse.

Sources and methods of conviction. Mathematical truth is not revealed, and its acceptance is not imposed by any authority.

Moreover, mathematical truth decidedly is not something that can be ascertained, as Justice Oliver Wendell Holmes put it, by "the majority vote of the nation that could lick all the others" (quoted from [Pe]), or by acceptance "in the competition of the market". In short, it is not a democratic value.

Ideally, the truth of a mathematical statement is ensured by a proof, and the ideal picture of a proof is a sequence of elementary arguments whose rules of formation are explicitly laid down before the proof even begins, and ideally are common for all proofs that have been devised and can be devised in future. The admissible starting points of proofs, "axioms", and terms in which they are formulated, should also be discussed and made explicit.

This ideal picture is so rigid that it can itself become the subject of mathematical study, which was actually performed and led to several remarkable discoveries, 
technically all related to the effects of merging language with metalanguage and self-referentiality.

Of course, the real life proofs are rendered in a peculiar mixture of a natural language, formulas, motivations, examples. They are much more condensed than imaginary formal proofs. The ways of condensing them are not systematic in any way. We are prone to mistakes, to taking on trust others' results that can be mistaken as well, and to relying upon authority and revelations from our teachers. (All of this should have been discussed together with the notion of "humans" which I have wisely avoided.)

Moreover, the discovery of truth may, and usually does, involve experimentation, nowadays vast and computer-assisted, false steps, sudden insights and all that which makes mathematical creativity so fascinating for its adepts.

One metaphor of proof is a route, which might be a desert track boring and unimpressive until one finally reaches the oasis of one's destination, or a foot path in green hills, exciting and energizing, opening great vistas of unexplored lands and seductive offshoots, leading far away even after the initial destination point has been reached.

[...] "mismanagement and grief": here you have that enormous distance between cause and effect covered in one line. Just as math preaches how to do it.

J. Brodsky. On "September 1, 1939" by W. H. Auden.

Mathematics is most visible to the general public when it is posits itself as an applied science, and in this role the notion of mathematical truth acquires distinctly new features. For example, our initial discussion of $\pi$ as an essentialy non-finitary ("irrational") real number becomes pointless; whenever $\pi$ enters any practical calculation, the first few digits are all that matters.

In a wider context than just applied science, mathematics can be fruitfully conceived as a toolkit containing powerful cognitive devices. I have argued elsewhere ([Ma1], [Ma2]) that these devices can be roughly divided into three overlapping domains: models, theories, and metaphors. Quoting from [Ma2],

"A mathematical model describes a certain range of phenomena qualitatively or quantitatively but feels uneasy pretending to be something more. 
From Ptolemy's epicycles (describing planetary motions, ca 150) to the Standard Model (describing interactions of elementary particles, ca 1960), quantitative models cling to the observable reality by adjusting numerical values of sometimes dozens of free parameters ( $\geq 20$ for the Standard Model). Such models can be remarkably precise.

Qualitative models offer insights into stability/instability, attractors which are limiting states tending to occur independently of initial conditions, critical phenomena in complex systems which happen when the system crosses a boundary between two phase states, or two basins of different attractors. [...]

What distinguishes a (mathematically formulated physical) theory from a model is primarily its higher aspirations. A modern physical theory generally purports that it would describe the world with absolute precision if only it (the world) consisted of some restricted variety of stuff: massive point particles obeying only the law of gravity; electromagnetic field in a vacuum; and the like. [...]

A recurrent driving force generating theories is a concept of a reality beyond and above the material world, reality which may be grasped only by mathematical tools. From Plato's solids to Galileo's "language of nature" to quantum superstrings, this psychological attitude can be traced sometimes even if it conflicts with the explicit philosophical positions of the researchers.

A (mathematical) metaphor, when it aspires to be a cognitive tool, postulates that some complex range of phenomena might be compared to a mathematical construction. The most recent mathematical metaphor I have in mind is Artificial Intelligence (AI). On the one hand, AI is a body of knowledge related to computers and a new, technologically created reality, consisting of hardware, software, Internet etc. On the other hand, it is a potential model of functioning of biological brains and minds. In its entirety, it has not reached the status of a model: we have no systematic, coherent and extensive list of correspondences between chips and neurons, computer algorithms and brain algorithms. But we can and do use our extensive knowledge of algorithms and computers (because they were created by us) to generate educated guesses about structure and function of the central neural system $[\ldots]$.

A mathematical theory is an invitation to build applicable models. A mathematical metaphor is an invitation to ponder upon what we know."

As an aside, let us note that George Lakoff's definition of poetic metaphors such as "love is a journey" in [La] is itself expressed as a mathematical metaphor using the characteristic Cantor-Bourbaki mental images and vocabulary: "More technically, 
the metaphor can be understood as a mapping (in the mathematical sense) from a source domain (in this case, journeys) to a target domain (in this case, love). The mapping is tightly structured. There are ontological correspondences, according to which entities in the domain of love (e. g. the lovers, their common goals, their difficulties, the love relationship, etc.) correspond systematically to entities in the domain of a journey (the travellers, the vehicle, destinations, etc.)."

When a mathematical construction is used as a cognitive tool, the discussion of truth becomes loaded with new meanings: a model, a theory or a metaphor must be true to a certain reality, more tangible and real than the Platonic "reality" of pure mathematics. In fact, philosophers of science routinely discussed truth precisely in this context. Karl Popper's vision of scientific theories in terms of falsifiability (vs verifiability) is quite appropriate in the context of highly mathematicised theories as well.

What I want to stress here, however, is one aspect of contemporary mathematical models which is historically very recent. Namely, models are more and more widely used as "black boxes" with hidden computerized input procedures, and oracular outputs prescribing behavior of human users.

Mary Poovey, discussing from this viewpoint financial markets, remarks in her insightful essay $[\mathrm{Po}]$ that what she calls "representations", basically computerized bookkeeping or the numbers a trader enters in a computer, tend to replace the actual exchange of cash or commodities. "This conflation of representation and exchange has all kinds of material effects, [...] for when representation can influence or take the place of exchanges, the values at stake become notional too: they can grow exponentially or collapse at the stroke of key".

In fact, actions of traders, banks, hedge funds and alike are to a considerable degree determined by the statistical models of financial markets encoded in the software of their computers. These models thus become a hidden and highly influential part of the actions, our computerized "collective unconscious". As such, they cannot even be judged according to the usual criteria of choosing models which better reflect the behavior of a process being modeled. They are part of any such process.

What becomes more essential than their empirical adequacy, is, for example, their stabilizing or destabilizing potential. Risk management assuming mild variability and small risks can collapse when a disaster occurs, ruining many participants of the game; risk management based upon models that use pessimistic "Lévy distributions" rather than omnipresent Gaussians paradoxically tends to flatten the shock waves and thus to avoid major disasters (cf. [MandHu]). 
5

There have been dramatic changes in the way in which the motion of the crowd is modeled in recent years.

R. Clemens, R. Hughes, in [ClHu].

When in the 20th century mathematicians got involved in heated discussions about the so called "Crisis in Foundations of Mathematics", several issues were intermingled.

Philosophically-minded logicians and professional philosophers were engaged with the nature and accessibility of mathematical truth (and reliability of our mental tools used in the process of acquiring it).

Logicists (finitists, formalists, intuitionists) were elaborating severe normative prescriptions trying to outlaw dangerous mental experiments with infinity, nonconstructivity and reductio ad absurdum.

For a working mathematician, when he/she is concerned at all, "foundations" is simply a general term for the historically variable set of rules and principles of organization of the body of mathematical knowledge, both existing and being created. From this viewpoint, the most influential foundational achievement in the 20th century was an ambitious project of the Bourbaki group, building all mathematics, including logic, around set-theoretical "structures" and making Cantor's language of sets a common vernacular of algebraists, geometers, probabilists and all other practitioners of our trade. These days, this vernacular, with all its vocabulary and ingrained mental habits, is being slowly replaced by the languages of category theory and homotopy theory and their higher extensions. Respectively, the basic "left-brain" intuition of sets, composed of distinguishable elements, is giving way to a new, more "right brain" basic intuition dealing with space-like and continuous primary images, both deformable and deforming.

In the Western ethnomathematics, truth is best understood as a central value, ever to be pursued, rather than anything achieved. Practical efficiency, authority, success in competition, faith, all other clashing values must recede in the mind of a mathematician when he or she sets down to do their job.

The most interesting intracultural interactions of mathematics such as symbolized by this conference are as well those that are not direct but rather proceed with the mediation of value systems. 


\section{Coda}

Every four years, mathematicians from all over the world meet at the International Congresses (ICM), to discuss whatever interesting developments happened recently in their domains of expertise. One of the traditions of these Congresses is a series of lectures for general public.

In 1998, our Congress met in Berlin, and Hans Magnus Enzensberger, the renowned poet and essayist, deeply interested in mathematics, spoke about "Zugbrücke außer Betrieb: die Mathematik im Jenseits der Kultur": the drawbridge to the castle of mathematics is out of service. The main concern of his talk was a deplorable lack of mathematical culture and communication between the general public and mathematicians, leading to alienation and mutual mistrust.

At the end of his talk ([Enz]) Enzensberger quotes an imaginary dialogue from [St], where a mathematician is chatting with a fictitional layman "Seamus Android".

"Mathematician: It's one of the most important discoveries of the last decade!

Android: Can you explain it in words ordinary mortals can understand?

Mathematician: Look, buster, if ordinary mortals could understand it, you wouldn't need mathematicians to do the job for you, right? You can't get a feeling for what's going on without understanding the technical details. How can I talk about manifolds without mentioning that the theorems only work if the manifolds are finite-dimensional para-compact Hausdorff with empty boundary?

Android: Lie a bit.

Mathematician: Oh, but I couldn't do that!

Android: Why not? Everybody else does."

And here I must play God and say to both Android and Mathematician: "Oh, no! Don't lie - because everybody else does."

\section{References}

[Bl] S. Blackburn. Truth and Ourselves: the Elusive Last Word. Keynote talk at the Balzan Symposium "Truth", May 2008.

[BoHa] L. Bovens, S. Hartmann. Bayesian Epistemology. Clarendon Press, Oxford, 2003.

$[\mathrm{ClHu}]$ R. C. Clemens, R. L. Hughes. Mathematical Modelling of a Mediaeval Battle: the Battle of Agincourt, 1415. Math. and Computers in Simulation, 64:2 (2004), 259-269. 
[Dau] J. W. Dauben. Georg Cantor. His Mathematics and Philosophy of the Infinite. Princeton University Press, 1990.

[DavHe] P. Davis, R. Hersh. The Mathematical Experience. Birkhäuser Boston, 1986.

[Enz] H. M. Enzensberger. Drawbridge Up. Mathematics - a Cultural Anathema. A. K. Peters, Natick, Mass., 1999.

[La] G. Lakoff. The Contemporary Theory of Metaphor. In: A. Ortony (ed.), Metaphor and Thought (2nd ed.). Cambridge Univ. Press, 1993.

[MandHu] B. Mandelbrot, R. Hudson. The (Mis)behavior of Markets: a Fractal View of Risk, Ruin and Rewards. Profile, 2005.

[Ma1] Yu. Manin. Mathematics as Metaphor. (Selected Essays, with Foreword by F. Dyson). American Math. Society, 2007.

[Ma2] Yu. Manin. Mathematical Knowledge: Internal, Social and Cultural Aspects. (Introductory Chapter to vol. 2 of "La Matematica", Einaudi, ed. by C. Bartocci and P. Odifreddi, reproduced in [Ma1], pp. 3-26). Preprint math.HO/0703427

[Pe] J. D. Peters. Courting the Abyss: Free Speech and the Liberal Tradition. Chicago, 2005.

[Po] M. Poovey. Can Numbers Ensure Honesty? Unrealistic expectations and the US accounting scandal. Notices of the AMS, vol. 50:1, Jan. 2003, pp. 27-35.

[St] I. Stewart. The Problems of Mathematics. Oxford Univ. Press, 1987. 